\title{
Phase transitions in nuclear matter
}

\author{
Jürgen Hofmann, Berndt Müller, and Walter Greiner \\ Institut für Theoretische Physik, Johann Wolfgang Goethe Universität, 6000 Frankfurt am Main, Germany
}

(Received 27 August 1979)

\begin{abstract}
A method for the description of spin-isospin phase transitions in nuclear matter is developed. It allows a complete description of the pion condensation phase transition in the framework of the Landau-Migdal Fermi liquid theory. The equation of the order parameter is derived and the condensation energy is calculated. We study the influence of pion condensation on the nuclear equation of state and the temperature dependence of pion condensation.
\end{abstract}

$$
\left[\begin{array}{cc}
\text { NUCLEAR STRUCTURE } & \begin{array}{c}
\text { Description of pion-condensed ground state by Green's } \\
\text { function technique. }
\end{array}
\end{array}\right.
$$

\section{INTRODUCTION}

Recent high energy heavy ion experiments ${ }^{1}$ point out the relevance of the investigation of the nuclear equation of state with respect to the possible occurrence of pion condensation,,$^{2}$ density isomer, ${ }^{3}$ or other abnormal states. The theoretical predictions of pion condensation (or more precisely, $\mathrm{p}-\mathrm{h}$ condensation) rely mainly on two different approaches: The propagator formalism ${ }^{4-6}$ in the normal ground state was applied to the determination of the phase transition point and to the study of its temperature dependence. ${ }^{7,8}$ This method is not able to describe the condensed phase beyond the transition point. The second approach is based on the chiral $\sigma$ model $^{9}$ of the $\pi-N$ interaction, which explicitly contains the possibility of pion condensation. The $\sigma$ model is in principle also applicable to the condensed phase but it is difficult to fix the value of the coupling constants: In the mean field approach the $\sigma$ model does not allow for a second minimum in the equation of state if its parameters are adjusted to describe normal nuclear matter. ${ }^{10}$

In the present paper we apply the Fermi liquid theory ${ }^{11}$ of nuclear matter to calculations of the properties of a $\mathrm{p}$-h-condensed nuclear phase. The parameters of this model are adjusted to the normal nuclear phase and can be smoothly extrapolated to the condensed phase. There is no need to introduce any additional assumptions.

\section{THE LANDAU-MIGDAL FERMI LIQUID THEORY OF PION CONDENSATION}

Up to now the use of Landau-Migdal Fermi liquid theory in the context of pion condensation calculations was restricted to take into account the short range nucleon-nucleon correlations. ${ }^{2}$ In the present paper we take a different point of view:
The Migdal ${ }^{12}$ interaction

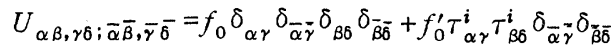

$$
\begin{aligned}
& +g_{0} \sigma_{\bar{\alpha} \bar{\gamma}}^{i} \sigma_{\bar{\beta} \bar{b}}^{i} \delta_{\alpha \gamma} \delta_{\beta \sigma}+g_{0}^{\prime} \tau_{\alpha \gamma}^{i} \tau_{\beta \bar{\sigma}}^{i} \sigma_{\bar{\alpha} \bar{\gamma}}^{k} \sigma_{\bar{B} \bar{b}}^{k}
\end{aligned}
$$

is taken as basic interaction for the particle-hole degree of freedom in nuclear matter. In Eq. (1) $\alpha, \beta, \gamma, \ldots$ denote the isospin and $\bar{\alpha}, \bar{\beta}, \bar{\gamma}, \ldots$ denote the spin indices. The coupling constants $f_{0}, \ldots, g_{0}^{\prime}$ contain the usual factor $C=\left(d f / d \epsilon_{F}\right)_{\rho=\rho_{0}}{ }^{-1}$ and are assumed to be momentum independent. Therefore $U$ corresponds to a zero range Skyrme interaction. It approximates the $\omega$ - and $\rho$-meson exchange part of the nuclear-nucleon interaction but does not take into account the long range pion exchange part. The implementation of one-pion exchange (OPE) will be described later. In our approximation we have replaced the single particle exchange by an effective interaction

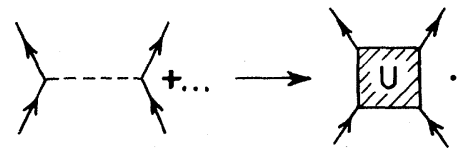

It must be stressed that the effective interaction (1) can be calculated from particle exchange..$^{13}$ Therefore also multiparticle exchange can be included in the effective coupling constants $f_{0}, \ldots, g_{0}^{\prime}$; i.e., by changing the coupling constants we may simulate all the essential features of the particlehole degree of freedom which are otherwise incorporated in pion condensation estimates only via lengthy calculations, e.g., $\Delta$-resonance formation, $\omega-\rho$ exchange, etc.

It is a common feature that a system of interacting particles in its "normal" ground state, suddenly becomes unstable and undergoes a phase transition to a new condensed state having completely different properties. It is well known that

(c) 1980 The American Physical Society 
the new ground state cannot be described by perturbation theory based on the old noncondensed ground state. ${ }^{14}$ The actual reason for this failure is the occurrence of anomalous propagation processes in the condensed state which describe spin or isospin flip. We therefore have to replace the usual Green's function $G_{i s}$ where $i$ denotes the isospin and $s$ the spin, by a $4 \times 4$ matrix of the following type:

$$
G_{\alpha \beta, \bar{\alpha} \bar{\beta}}=\left[\begin{array}{llll}
G_{p \uparrow p \uparrow} & G_{p \uparrow p \downarrow} & G_{p \uparrow n \uparrow} & G_{p \uparrow n \downarrow} \\
G_{p \downarrow p \uparrow} & G_{p \downarrow p \downarrow} & G_{p \downarrow n \uparrow} & G_{p \downarrow n \downarrow} \\
G_{n \uparrow p \uparrow} & G_{n \uparrow p \downarrow} & G_{n \uparrow n \uparrow} & G_{n \uparrow n \downarrow} \\
G_{n \downarrow p \uparrow} & G_{n \downarrow p \downarrow} & G_{n \downarrow n \uparrow} & G_{n \downarrow n \downarrow}
\end{array}\right],
$$

where, e.g., $G_{p \downarrow n \uparrow}=\left\langle\phi_{0}\left|T\left\{c_{p \downarrow} c_{n \uparrow}^{\dagger}\right\}\right| \phi_{0}\right\rangle$ propagates a neutron with spin up into a proton with spin down. It is obvious that in the normal noncondensed ground state only the diagonal matrix elements of (3) are nonzero. As soon as condensation occurs, anomalous propagation processes yield nonzero off-diagonal matrix elements for (3).

For the matrix propagator (3) we use the following ansatz:

$$
\begin{aligned}
G_{\alpha \beta, \bar{\alpha} \bar{\beta}}(\overrightarrow{\mathrm{k}}, \omega)=\frac{1}{4} & \left\{g(\overrightarrow{\mathrm{k}}, \omega) \delta_{\alpha \beta} \delta_{\bar{\alpha} \bar{\beta}}+\delta_{\alpha \beta} \vec{\sigma}_{\bar{\alpha} \bar{\beta}} \cdot \overrightarrow{\mathrm{S}}(\overrightarrow{\mathrm{k}}, \omega)\right. \\
& +\vec{\tau}_{\alpha \beta} \delta_{\bar{\alpha} \bar{\beta}} \overrightarrow{\mathrm{T}}(\overrightarrow{\mathrm{k}}, \omega) \\
& \left.+\left[\vec{\tau}_{\alpha \beta} \cdot \overrightarrow{\mathrm{T}}(\overrightarrow{\mathrm{k}}, \omega)\right]\left[\vec{\sigma}_{\bar{\alpha} \bar{\beta}} \cdot \overrightarrow{\mathrm{S}}(\overrightarrow{\mathrm{k}}, \omega)\right]\right\} .
\end{aligned}
$$

The unknown functions $g(\vec{k}, \omega), \overrightarrow{\mathrm{S}}(\overrightarrow{\mathrm{k}}, \omega), \overrightarrow{\mathrm{T}}(\overrightarrow{\mathrm{k}}, \omega)$ are determined from the solution of the Dyson equation for $G_{\alpha \beta, \bar{\alpha} \bar{\beta}}(\overrightarrow{\mathrm{k}}, \omega)$. The various components of the Green's function are related to physical observables in the following way ${ }^{16}$ :

$$
\begin{aligned}
& \rho(\overrightarrow{\mathrm{k}})=-i \int \frac{d \omega}{2 \pi} g(\overrightarrow{\mathrm{k}}, \omega) e^{-i \omega \delta_{-}}, \\
& \Sigma_{i}(\overrightarrow{\mathrm{k}})=-i \int \frac{d \omega}{2 \pi} S_{i}(\overrightarrow{\mathrm{k}}, \omega) e^{-i \omega \delta_{-}}, \\
& t_{i}(\overrightarrow{\mathrm{k}})=-i \int \frac{d \omega}{2 \pi} T_{i}(\overrightarrow{\mathrm{k}}, \omega) e^{-i \omega \delta_{-}}, \\
& p_{i k}(\overrightarrow{\mathrm{k}})=-i \int \frac{d \omega}{2 \pi} T_{i}(\overrightarrow{\mathrm{k}}, \omega) S_{k}(\overrightarrow{\mathrm{k}}, \omega) e^{-i \omega \delta_{-}}, \quad \delta_{-}=\lim _{t \rightarrow 0} t
\end{aligned}
$$

where $\rho(\overrightarrow{\mathbf{k}})$ is the nucleon density in momentum space and $\Sigma_{i}, t_{i}$, and $p_{i k}$ are the components of the spin-, isospin-, and spin-isospin densities, respectively. To obtain the Dyson equation, we calculate the Hartree-Fock self-energy with the interaction (1) and the Green's function (4). It is given by

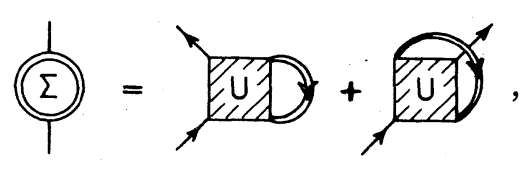

where the direct part $\Sigma_{1}$ is

$$
\begin{aligned}
\Sigma_{(1)_{\alpha \gamma}, \bar{\alpha} \bar{\gamma}}=-i \int \frac{d^{3} k}{(2 \pi)^{3}} \frac{d \omega}{2 \pi} \sum_{\beta \bar{\beta} \bar{\delta} \bar{\delta}} & U_{\alpha \beta, \gamma \bar{\delta} ; \bar{\alpha} \bar{\beta}, \bar{\gamma} \bar{\delta}} \\
& \times G_{\delta \beta, \bar{\delta} \bar{\beta}}(\overrightarrow{\mathrm{k}}, \omega) e^{-i \omega 0^{-}}
\end{aligned}
$$

and the exchange part

$$
\begin{aligned}
\Sigma_{(2) \alpha \gamma, \bar{\alpha} \bar{\gamma}}=i \int \frac{d^{3} k}{(2 \pi)^{3}} \frac{d \omega}{2 \pi} \sum_{\beta \bar{\beta} \bar{\delta} \bar{\delta}} & U_{\alpha \beta, \bar{\gamma} \gamma \bar{\alpha} \bar{\alpha} \overline{\bar{\delta}} \bar{\gamma}} \\
& \times G_{6 \beta, \bar{\delta} \bar{\beta}}(\overrightarrow{\mathrm{k}}, \omega) e^{-i \omega 0^{-}} .
\end{aligned}
$$

A straightforward calculation yields

$$
\begin{aligned}
\Sigma_{\alpha \gamma, \bar{\alpha} \bar{\gamma}}^{H F}= & F_{1} \rho \delta_{\alpha \gamma} \delta_{\bar{\alpha} \bar{\gamma}}+F_{2} \delta_{\alpha \gamma} \sigma_{\alpha \bar{\alpha}}^{i} \Sigma_{2} \\
& +F_{3} \tau_{\alpha \gamma}^{i} t_{i} \delta_{\bar{\alpha} \bar{\gamma}}+F_{4} \tau_{\alpha \gamma}^{i} \sigma_{\alpha \bar{\gamma}}^{k} p_{i k},
\end{aligned}
$$

where $\rho, \Sigma_{i}, t_{i}$, and $p_{i k}$ are the quantities (5) integrated with respect to $k$ and

$$
\begin{aligned}
& F_{1}=\frac{1}{4}\left(3 f_{0}-f_{0}^{\prime}-g_{0}-g_{0}^{\prime}\right), \\
& F_{2}=\frac{1}{4}\left(5 g_{0}-f_{0}-f_{0}^{\prime}+g_{0}^{\prime}\right), \\
& F_{3}=\frac{1}{4}\left(5 f_{0}^{\prime}-f_{0}-g_{0}+g_{0}^{\prime}\right), \\
& F_{4}=\frac{1}{4}\left(3 g_{0}^{\prime}-f_{0}-f_{0}^{\prime}+g_{0}\right) .
\end{aligned}
$$

The Dyson equation is

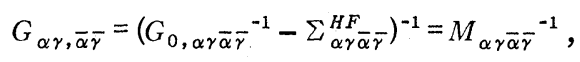

where

$$
\begin{aligned}
M_{\alpha \gamma \bar{\alpha} \bar{\gamma}}= & F \delta_{\alpha \gamma} \delta_{\bar{\alpha} \bar{\gamma}}-F_{2} \delta_{\alpha \gamma} \sigma \frac{i}{\alpha \bar{\gamma}} \Sigma_{i} \\
& -F_{3} \tau_{\alpha \gamma}^{i} t_{i} \delta_{\bar{\alpha} \bar{\gamma}}-F_{4} \tau_{\alpha \gamma}^{i} \sigma_{\bar{\alpha} \bar{\gamma}} p_{i k}
\end{aligned}
$$

with $F=\omega-\left(\epsilon_{k}-\mu\right)-F_{1} \rho+i \delta$. The free propagator is diagonal, i.e.,

$$
G_{0, \alpha \gamma \bar{\alpha} \bar{\gamma}}^{-1}=\Omega \delta_{\alpha \gamma} \delta_{\bar{\alpha} \bar{\gamma}},
$$

where

$$
\Omega=\omega-\left(\epsilon_{k}-\mu\right)+i \delta .
$$

As we are mainly interested in the pion related spin-isospin degree of freedom, we neglect the pure spin and isospin part in (12), i.e., we put $F_{2}=F_{3}=0$. Now (12) can be inverted and we obtain

$$
g(k, \omega)=\frac{4 \Omega-\alpha \rho}{\left(\Omega-\frac{1}{4} \alpha \rho+\frac{1}{4} \beta p\right)\left(\Omega-\frac{1}{4} \alpha \rho-\frac{1}{4} \beta p\right)}
$$

and

$$
P(k, \omega)=\frac{-\beta}{\left(\Omega-\frac{1}{4} \alpha \rho+\frac{1}{4} \beta p\right)\left(\Omega-\frac{1}{4} \alpha \rho-\frac{1}{4} \beta p\right)} p
$$


with

$$
\beta=f_{0}-f_{0}^{\prime}-g_{0}-3 g_{0}^{\prime}
$$

and

$$
\alpha=3 f_{0}-f_{0}^{\prime}-g_{0}-g_{0}^{\prime} .
$$

We have assumed that only $p=p_{z z}$ is different from zero which corresponds to the usual assumption that the pion condensate is described by a plane wave in $z$ direction in the infinite system.

Introducing the quasiparticle energies

$$
\omega_{ \pm}(k)=\epsilon_{k}-\frac{1}{4} \alpha \rho \mp \frac{1}{4} \beta p,
$$

we may define quasiparticle propagators

$$
g_{ \pm}(k, \omega)=\left[\omega-\left(\omega_{ \pm}-\mu\right)+i \delta J^{-1},\right.
$$

allowing us to rewrite (15) and (16) as

$$
\begin{aligned}
& g(k, \omega)=2\left[g_{+}(k, \omega)+g_{-}(k, \omega)\right], \\
& P(k, \omega)=2\left[g_{+}(k, \omega)-g_{-}(k, \omega)\right] .
\end{aligned}
$$

Inserting this into Eq. (5) we obtain after integration with respect to $\omega$

$$
\begin{aligned}
& \rho=2 \int \frac{d^{3} k}{(2 \pi)^{3}}\left[\rho_{+}(k)+\rho_{-}(k)\right], \\
& p=2 \int \frac{d^{3} k}{(2 \pi)^{3}}\left[\rho_{+}(k)-\rho_{-}(k)\right],
\end{aligned}
$$

where

$$
\rho_{ \pm}(k)= \begin{cases}1, & k<k_{F}^{ \pm} \\ 0, & k>k_{F}^{ \pm}\end{cases}
$$

and the quasiparticle Fermi momentum is

$$
k_{F}^{ \pm}=\left[2 m\left(\mu-\frac{1}{4} \alpha \rho \pm \frac{1}{4} \beta p\right)\right]^{1 / 2} .
$$

We now may perform the $k$ integration to obtain

$$
\begin{gathered}
\rho=\frac{\rho}{2 \epsilon_{F}^{3 / 2}}\left[\left(\mu-\frac{1}{4} \alpha \rho+\frac{1}{4} \beta \rho M_{\pi}\right)^{3 / 2}\right. \\
\left.+\left(\mu-\frac{1}{4} \alpha \rho-\frac{1}{4} \beta \rho M_{\pi}\right)^{3 / 2}\right], \\
M_{\pi}=\frac{1}{2 \epsilon_{F}^{3 / 2}}\left[\left(\mu-\frac{1}{4} \alpha \rho+\frac{1}{4} \beta \rho M_{\pi}\right)^{3 / 2}\right. \\
\left.-\left(\mu-\frac{1}{4} \alpha \rho-\frac{1}{4} \beta \rho M_{\pi}\right)^{3 / 2}\right] .
\end{gathered}
$$

Here is the Fermi energy

$$
\epsilon_{F}=\left(\frac{3 \pi^{2}}{2}\right)^{2 / 3} \frac{\rho^{2 / 3}}{2 m}
$$

and we have defined the spin-isospin polarization as

$$
M_{\pi}=p / \rho \text {. }
$$

By eliminating $\mu$ from (25) and (24) we obtain the self-consistent equation for $M_{\pi}$ :

$$
\frac{\beta \rho}{2 \epsilon_{F}}=\frac{1}{M_{\pi}}\left[\left(1+M_{\pi}\right)^{2 / 3}-\left(1-M_{\pi}\right)^{2 / 3}\right] \text {. }
$$

The actual value of $M_{\pi}$ depends only on the effective coupling constant

$$
\frac{\beta \rho}{2 \epsilon_{F}}=\frac{1}{3} \tilde{\beta}\left(\frac{\rho}{\rho_{0}}\right)^{1 / 3},
$$

where $\tilde{\beta}=f_{0}-f_{0}^{\prime}-g_{0}-3 g_{0}^{\prime}$ is now dimensionless; $\rho$ is the actual nuclear density and $\rho_{0}$ the nuclear equilibrium density. It is obvious from (28) that $M_{\pi}=0$ as long as $\beta \rho / 2 \epsilon_{F}<\frac{4}{3}$. Equation (29) allows a solution with $M_{\pi} \neq 0$ only if $\beta \rho / 2 \epsilon_{F} \geqslant \frac{4}{3}$. Nevertheless $M_{\pi}=0$ is a possible solution also beyond this critical point. To check whether $M_{\pi} \neq 0$ is indeed the solution beyond $\beta \rho /{ }_{2} \epsilon_{F}=\frac{4}{3}$ we have to calculate the total energy ${ }^{15}$

$$
E=-\frac{i}{2} \int \frac{d^{3} k}{(2 \pi)^{3}} \frac{d \omega}{2 \pi}\left(\frac{k^{2}}{2 m}+\omega+\mu\right) \operatorname{Tr} G(k, \omega) e^{-i \omega 0^{-}} .
$$

As $\operatorname{Tr} G(k, \omega)=g(k, \omega)=2\left[g_{+}(k, \omega)+g_{-}(k, \omega)\right]$ we obtain with (18)

$$
\begin{aligned}
E= & 2 \int \frac{d^{3} k}{(2 \pi)^{3}} \frac{k^{2}}{2 m}\left[\rho_{+}(k)+\rho_{-}(k)\right] \\
& +\frac{1}{2} \alpha \int \frac{d^{3} k}{(2 \pi)^{3}} \frac{d^{3} p}{(2 \pi)^{3}}\left[\rho_{+}(p)+\rho_{-}(p)\right]\left[\rho_{+}(k)+\rho_{-}(k)\right] \\
& -\frac{1}{2} \beta \int \frac{d^{3} k}{(2 \pi)^{3}} \frac{d^{3} p}{(2 \pi)^{3}}\left[\rho_{+}(p)-\rho_{-}(p)\right]\left[\rho_{+}(k)-\rho_{-}(k)\right],
\end{aligned}
$$

where we have used $\rho=\int d^{3} p /(2 \pi)^{3}\left[\rho_{+}(p)+\rho_{-}(p)\right]$, etc. Expressing $k_{F}^{ \pm}=\left(2 m \epsilon_{F}\right)^{1 / 2}\left(1 \pm M_{\pi}\right)^{1 / 3}$ we obtain after integration

$$
\begin{aligned}
\epsilon= & \frac{3}{10}\left[\left(1+M_{\pi}\right)^{5 / 3}+\left(1-M_{\pi}\right)^{5 / 3}\right]+\frac{1}{4}\left(\alpha \rho / 2 \epsilon_{F}\right) \\
& -\frac{1}{4}\left(\beta \rho / 2 \epsilon_{F}\right) M_{\pi}^{2},
\end{aligned}
$$

where we have introduced

$$
\epsilon=E / \epsilon_{F} N \text {. }
$$

For small polarization $M_{\pi} \ll 1$ the Taylor series up to second order reads:

$$
\epsilon \approx \frac{3}{5}+\frac{1}{4}\left(\alpha \rho / 2 \epsilon_{F}\right)+\frac{1}{4}\left(\frac{4}{3}-\beta \rho / 2 \epsilon_{F}\right) M_{\pi}^{2} .
$$

It is obvious, that for $\beta \rho / 2 \epsilon_{F}>\frac{4}{3}$ the state with $M_{\pi} \neq 0$ has lower energy, i.e., condensation really occurs if $\beta>0$, i.e., $\tilde{\beta}>0$.

If we disregard the spin-spin and isospin-isospin interaction part, we obtain $\tilde{\beta}=f_{0}-3 g_{0}^{\prime}$. For the empirical values ${ }^{12} f_{0}=1 \pm 0.2$ and $g_{0}^{\prime} \simeq 0.7 \pm 0.2$ we find $\tilde{\beta}<0$. Ther efore no condensation can occur with normal Landau-Migdal parameters. Since we have taken into account only the zero range part of the interaction, which is repulsive, this result is easily understood. As the finite momentum pion-nucleon interaction is the relevant degree of freedom which influences the spin-isospin interaction constant $g_{0}$, we have to use a "renormalized" $g_{0}$ taking into account one-pion exchange. This has been investi- 


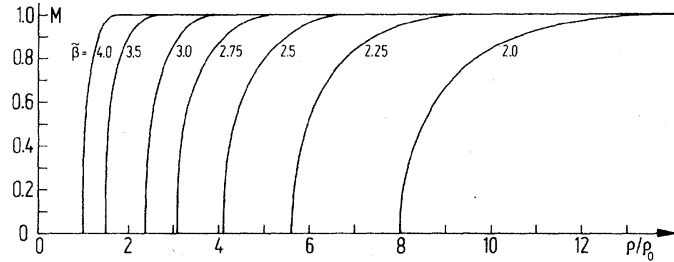

FIG. 1. The spin-isospin polarization $M_{\text {r }}$ as function of the nuclear density $\rho$ is shown for the different values of $\tilde{\beta}$.

gated on the basis of a Reid soft core model. ${ }^{17}$ There $g_{0}^{\prime}$ attains a minimal value of $g_{0}^{\prime} \sim-0.6$ at pion momenta of $k_{\pi} \sim(2-3) m_{\pi}$. Hence $\tilde{\beta} \simeq 3 \pm 0.5$ is the range of relevant physical parameters. The critical densities can now be obtained from (30) as

$$
\rho_{\mathrm{cr}} / \rho_{0}=(4 / \tilde{\beta})^{3} \text {, }
$$

i.e., $\rho_{\text {cr }} / \rho_{0} \sim 3 \pm 1.5$ for the actual values of $\tilde{\beta}$. The spin-isospin polarization $M_{\pi}(\rho)$ is shown in Fig. 1 for different values of $\tilde{\beta}$. The width of the phase transition is easily calculated to be

$$
\Delta \rho=\frac{\rho\left(M_{\pi}=1\right)}{\rho_{0}}-\frac{\rho_{c r}}{\rho_{0}}=\frac{44}{\widetilde{\beta}^{3}} .
$$

The strong dependence of $\Delta \rho$ and $\rho_{\mathrm{cr}} / \rho_{0}$ on $\tilde{\beta}$ is shown in Fig. 2. For $\tilde{\beta}=3$ the width of the phase transition $\Delta \rho \sim \rho_{0}$ whereas for $\tilde{\beta}=1 \Delta \rho \sim 44 \rho_{0}$, i.e., an extremely broad phase transition occurs at a very high density. Beyond the critical density we are interested in the condensation energy per particle, as a fraction of the Fermi energy:

$$
\epsilon_{\text {cond }}=\epsilon\left(M_{\pi}=0\right)-\epsilon\left(M_{\pi} \neq 0\right)
$$

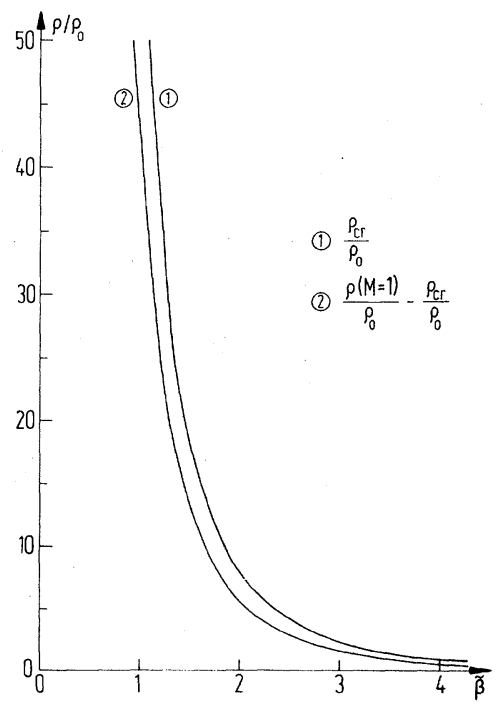

FIG. 2. The critical density $\rho_{\mathrm{cr}}$ and the density spreading $\Delta \rho$ of the phase transition strongly depend on $\tilde{\beta}$.

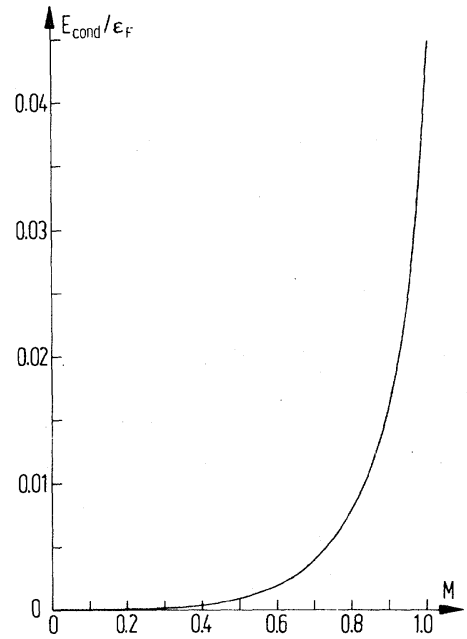

FIG. 3. The condensation energy in units of the (density dependent) Fermi energy is a unique function of $M_{\pi}$ independent of $\tilde{\beta}$.

or

$$
\begin{aligned}
\epsilon_{\text {cond }}= & \frac{3}{5}-\frac{3}{10}\left[\left(1+M_{\pi}\right)^{5 / 3}+\left(1-M_{\pi}\right)^{5 / 3}\right] \\
& +\frac{1}{4}\left(\beta \rho / 2 \epsilon_{F}\right) M_{\pi}{ }^{2} .
\end{aligned}
$$

By means of Eq. (28) we reexpress $\epsilon_{\text {cond }}$ as a function of $M_{\pi}$ alone:

$$
\begin{aligned}
\epsilon_{\text {cond }}= & \frac{3}{5}-\frac{3}{10}\left[\left(1+M_{\pi}\right)^{5 / 3}+\left(1-M_{\pi}\right)^{5 / 3}\right] \\
& +\frac{1}{4} M_{\pi}\left[\left(1+M_{\pi}\right)^{2 / 3}-\left(1-M_{\pi}\right)^{2 / 3}\right] .
\end{aligned}
$$

The maximal energy gain is obtained for $M_{\pi}=1$, i.e.,

$$
\epsilon_{\text {cond }}\left(M_{\pi}=1\right) \simeq 0.045 \text {. }
$$

This means that the maximum energy gain is less than $5 \%$ of the density dependent Fermi energy.

The dependence of $\epsilon_{\text {cond }}$ on $M_{\pi}$ is shown in Fig. 3, whereas Fig. 4 shows the actual condensation energy for different values of $\tilde{\beta}$. It shows that in

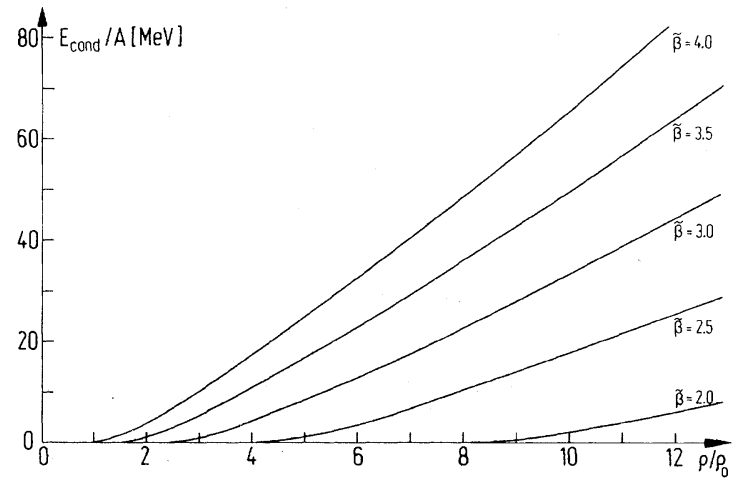

FIG. 4. The condensation energy per particle as function of the density is shown for different $\tilde{\beta}$. 


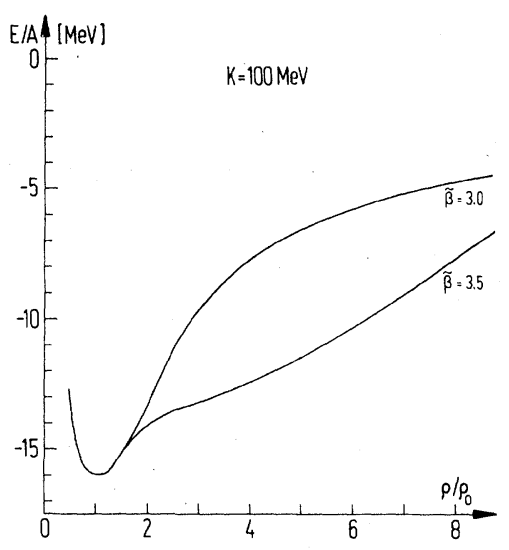

FIG. 5. Shows the nuclear equation of state for $K=100$ $\mathrm{MeV}$ under the influence of pion condensation with $\tilde{\beta}=3$ and $\tilde{\beta}=3.5$.

the relevant density region the condensation energy per particle is of the order of $10 \mathrm{MeV}$ for $\tilde{\beta}=3$. To investigate the relevance of spin-isospin condensation for the nuclear equation of state, we add the condensation energy onto a normal nuclear equation of state, ${ }^{18}$ e.g.,

$$
\frac{E}{A}=\frac{K}{18 \rho \rho_{0}}\left(\rho-\rho_{0}\right)^{2}+B_{0},
$$

where $K$ is the compression constant and $B_{0} \sim-16$ $\mathrm{MeV}$ the binding energy of normal nuclear matter. This method is certainly not self-consistent in the sense that we do not describe the normal state and the condensed state on an equal level. Nevertheless it may give an idea of the possible influence of pion condensation on the equation of state. The energy per particle is given as

$$
\frac{E}{A}\left(M_{\pi} \neq 0\right)=\frac{K}{18 \rho \rho_{0}}\left(\rho-\rho_{0}\right)^{2}+B_{0}+\epsilon_{\text {cond }} \epsilon_{F} .
$$

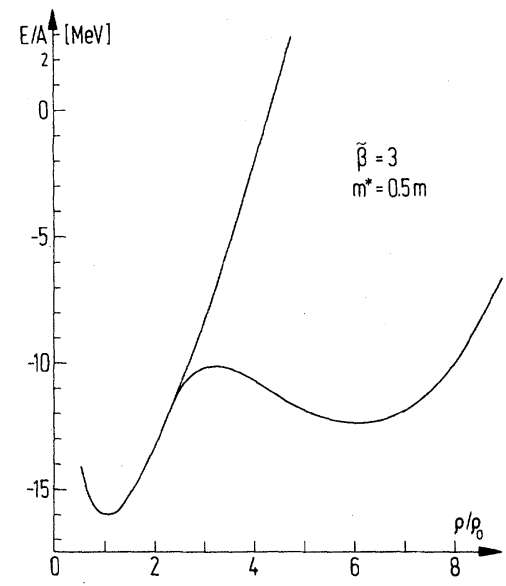

FIG. 6. An effective mass of $m^{*}=0.5 \mathrm{~m}$ causes a second minimum in the equation of state for, $\tilde{\beta}=3$.

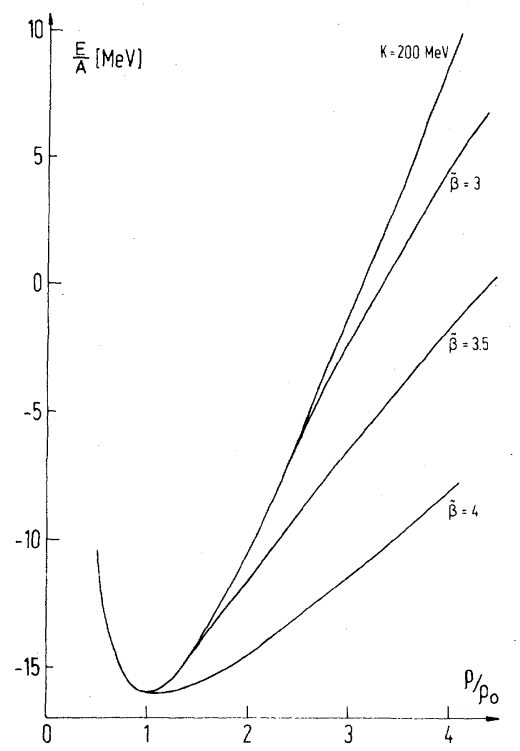

FIG. 7. For a compression constant $K=200 \mathrm{MeV}$ even no van der Waals behavior shows up in the equation of state.

The actual nuclear equation of state with pion condensation is shown in Figs. 5-7 for different values of $K$ and $\tilde{\beta}$. For small $K(K \sim 100 \mathrm{MeV})$ and large $\tilde{\beta}(\tilde{\beta}>3)$ the normal equation of state is strongly modified. At least an inflection point occurs which yields a noticeable modification in the behavior of nuclear matter in shock wave dynamics. ${ }^{1}$ If we introduce an effective nucleon mass $m^{*}$, the condensation energy increases. We therefore obtain a reasonable second minimum in $E / A(\rho)$ (Fig. 6). The amount of influence the pioncondensed mode exerts on the equation of state strongly depends on the compression constant $K$ of normal nuclear matter. Figure 7 shows that for $K=200 \mathrm{MeV}$ the compression energy rises much faster than $\epsilon_{\text {cond. }}$. The influence of pion condensation therefore shows up only as a slight modification in the sense of an effective compression constant $K<200 \mathrm{MeV}$.

\section{THE TEMPERATURE DEPENDENCE}

Another interesting question in this context is the stability of a pion condensate at high temperatures occurring in heavy ion collisions ${ }^{1}$ at relativistic energies. Previous calculations ${ }^{7}$ utilizing the pion propagator formalism show that a pion condensate may be stable up to very high temperature and is not destroyed by the thermal fluctuations. The method put forward in the present paper can easily be extended to temperature dependent matrix propagators. ${ }^{16}$ Instead of (13) the free propagator is 


$$
g_{0}\left(k, \omega_{n}\right)=\left(i \omega_{n}-\epsilon_{k}+\mu\right)^{-1},
$$

where the discrete frequencies

$$
\omega_{n}=(2 n+1) \pi k T
$$

and the ansatz (4) is modified to

$$
\begin{gathered}
g\left(k, \omega_{n}\right)=\frac{1}{4}\left\{g\left(k, \omega_{n}\right)+\vec{\sigma} \cdot \overrightarrow{\mathrm{S}}\left(k, \omega_{n}\right)+\vec{\tau} \cdot \overrightarrow{\mathrm{T}}\left(k, \omega_{n}\right)\right. \\
\left.+\left[\vec{\tau} \cdot \overrightarrow{\mathrm{T}}\left(k, \omega_{n}\right)\right]\left[\vec{\sigma} \cdot \overrightarrow{\mathrm{S}}\left(k, \omega_{n}\right)\right]\right\} .
\end{gathered}
$$

The integrals over frequency in Eqs. (5) are replaced by sums:

$$
\rho(k)=-\frac{1}{\beta} \sum_{n=-\infty}^{\infty} g\left(k, \omega_{n}\right) e^{i \omega_{n} 0^{+}}, \text {etc } .
$$

The essential modification connected with the temperature dependence is the replacement of

$$
\rho(k)=\theta\left(k-k_{F}\right), \quad T=0
$$

by

$$
\rho(k)=\frac{1}{e^{\beta\left(\epsilon_{k}-\mu\right)}+1},
$$

where $\beta=1 / k T$. Therefore (21) and (22) are replaced by

$$
\begin{aligned}
& \rho=2 \int_{0}^{\infty} \frac{d^{3} k}{(2 \pi)^{3}}\left(\frac{1}{e^{\beta\left(\omega_{+}-\mu\right)}+1}+\frac{1}{e^{\beta\left(\omega_{-}-\mu\right)}+1}\right), \\
& p=2 \int_{0}^{\infty} \frac{d^{3} k}{(2 \pi)^{3}}\left(\frac{1}{e^{\beta\left(\omega_{+}-\mu\right)}+1}-\frac{1}{e^{\beta\left(\omega_{-}-\mu\right)}+1}\right),
\end{aligned}
$$

where $\omega_{ \pm}=\epsilon_{k}+\frac{1}{4} \alpha \rho \mp \frac{1}{4} \beta p$. The Equations (49) and (50) may be rewritten as

$$
\rho=2\left(\mathcal{F}_{+}+\mathfrak{F}_{-}\right) ; \quad p=2\left(\mathcal{F}_{+}-\mathcal{F}_{-}\right),
$$

where

$$
\mathcal{F}_{+}=\frac{(2 m)^{3 / 2}}{(2 \pi)^{2}} \int_{0}^{\infty} \frac{\sqrt{\epsilon} d \epsilon}{e^{(1 / T)\left(\epsilon-\mu_{+}\right)}+1}
$$

and

$$
\mu_{ \pm}=\mu-\frac{1}{4} \alpha \rho \pm \frac{1}{4} \beta p .
$$

The integration over $\epsilon$ can be performed by series expansion $^{19}$

$$
\begin{aligned}
\mathcal{F}_{ \pm} \simeq \frac{(2 m)^{3 / 2}}{(2 \pi)^{2}} & \left(\frac{2}{3} \mu_{ \pm}^{3 / 2}+\frac{\pi^{2}}{12} \mu_{ \pm}{ }^{-1 / 2} T^{2}\right. \\
& \left.+\frac{7 \pi^{4}}{960} \mu_{ \pm}{ }^{-5 / 2} T^{4}+\cdots\right) .
\end{aligned}
$$

Introducing $M_{\pi}=p / \rho$ as previously, we obtain from (51) for $T \ll \epsilon_{F}$

$$
\begin{aligned}
& 1+M_{\pi}=\frac{1}{\tilde{\mu}_{+}^{1 / 2}}\left(\tilde{\mu}_{+}^{2}+\frac{\pi^{2}}{8} \tilde{T}^{2}\right), \\
& 1-M_{\pi}=\frac{1}{\tilde{\mu}_{-}^{1 / 2}}\left(\tilde{\mu}_{-}^{2}+\frac{\pi^{2}}{8} \tilde{T}^{2}\right),
\end{aligned}
$$

where $\tilde{\mu}_{ \pm}=\mu_{ \pm} / \epsilon_{F}$ and $\tilde{T}=T / \epsilon_{F} . \quad \tilde{\mu}_{ \pm}$can be elimi-

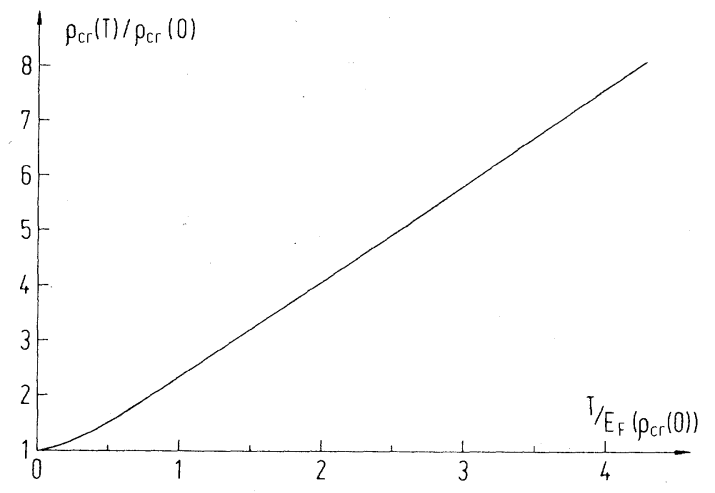

FIG. 8. The ratio of critical densities $\rho_{\text {cr }}$ at $T=0$ and $T \neq 0$ as function of the temperature in units of the Fermi energy at $\rho_{\mathrm{cr}}(T=0)$ is shown.

nated from (55) yielding again the spin-isospin polarization $M_{\pi}$ for $T \neq 0$.

The critical density $\rho_{\mathrm{cr}}(T)$ can be calculated exactly by defining $\mu^{\prime}=\mu-\frac{1}{4} \alpha \rho$. The condensation point is marked by the condition that (50) allows a solution with $p \neq 0$ for the first time. With $\tilde{\rho}(x)=\left(e^{\beta\left(x-\mu^{\prime}\right)}+1\right)^{-1}$ we get from (49) and (50) by differentiating with respect to $p$ :

$$
\begin{aligned}
& 1=-\frac{\beta}{4} \frac{(2 m)^{3 / 2}}{\pi^{2}} \int_{0}^{\infty} \sqrt{\epsilon} d \epsilon \frac{\partial \tilde{\rho}}{\partial \epsilon}\left[\mu^{\prime}\left(\rho_{c}\right)\right], \\
& \rho_{c}=\frac{(2 m)^{3 / 2}}{\pi^{2}} \int_{0}^{\infty} \sqrt{\epsilon} d \epsilon \tilde{\rho}\left[\mu^{\prime}\left(\rho_{c}\right)\right] .
\end{aligned}
$$

After some formal manipulations one obtains the boundary between the normal and condensed phase in parametric representation:

$$
\frac{\rho_{\mathrm{cr}}(T)}{\rho_{\mathrm{cr}}(0)}=\frac{3}{2} \frac{I_{0}(a)}{\left[I_{1}(a)\right]^{3}}
$$

and

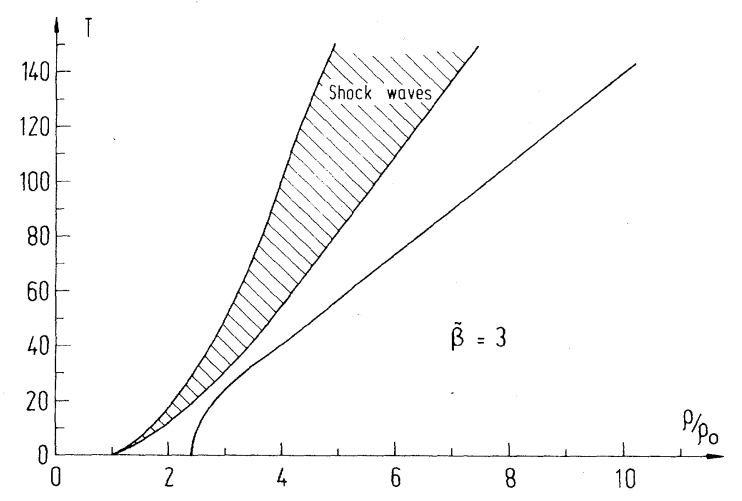

FIG. 9. The connection between the critical density $\rho_{\text {cr }}$ and the temperature is shown $f_{\text {cr }} \tilde{\beta}=3$. The dashed area denotes the temperature and density region obtained from shock wave calculations (Ref. 1). 


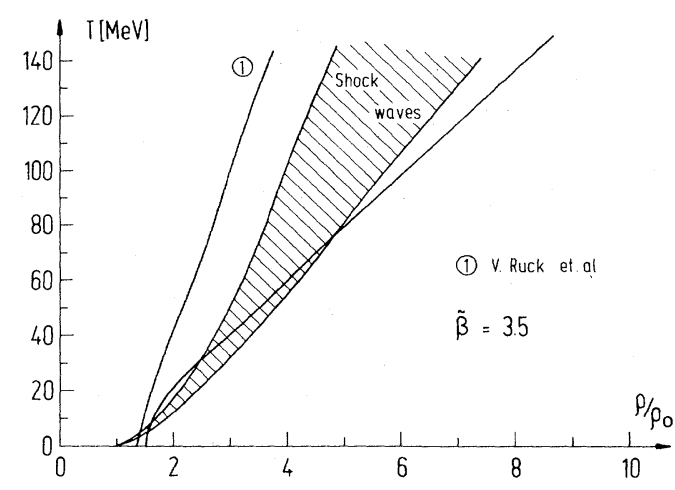

FIG. 10. Same as Fig. 9 for $\tilde{\beta}=3.5$. 1 denotes a previous calculation by Ruck et al. (Ref. 7).

$$
\frac{T}{\epsilon_{F}^{c r}}=\left[I_{1}(a)\right]^{-2}
$$

where

$$
\begin{aligned}
& I_{0}(a)=\int_{0}^{\infty} \frac{x^{1 / 2} d x}{e^{x-a}+1}, \\
& I_{1}(a)=\frac{d}{d a} I_{0}(a)=\int_{0}^{\infty} \frac{x^{1 / 2} e^{x-a} d x}{\left(e^{x-a}+1\right)^{2}},
\end{aligned}
$$

and $a=\mu^{\prime} / T$. $\epsilon_{F}^{c r}$ is the Fermi energy at the critical density $\rho_{\mathrm{cr}}^{(0)}$ and $\rho_{\mathrm{cr}}(0)$ is the critical density at $T=0$.

The resulting curve is shown in Fig. 8. Figures 9 and 10 show the actual critical density $\rho_{\mathrm{cr}}$ and the corresponding temperature for $\tilde{\beta}=3$ and $\tilde{\beta}=3.5$. The shock wave temperatures ${ }^{1}$ are indicated by a dashed region. It is obvious that the pion condensation stability curves are crossing the shock wave region or even lie below. In view of the rather schematic nature of our model there is certain chance for a pion condensate to survive the temperatures occurring in relativistic heavy ion collisions. This is in qualitative, although not in quantitative, agreement with previous calculations. ${ }^{7}$

\section{CONCLUSIONS}

In this paper we have presented a model of pion condensation allowing for a description beyond the phase transition point without giving up analytical tractability. It also allows to investiage the tem- perature dependence of the phase transition. The momentum dependence of the pion-nucleon interaction was taken into account phenomenologically by a suitable adjustment of the spin-isospin parameter $g_{0}^{\prime}$. In principle, the $k$ dependence of the interaction could be incorporated from the beginning but at the cost of losing the analytical solubility of the model. It will be subject of a future numerical investigation. The adjustment of $g_{0}^{\prime}$ leads to a prediction of the critical density $\rho_{\text {cr }}(T=0) \sim 3 \rho_{0}$ which agrees with previous calculations. ${ }^{2}$ As the condensation energy is of the order of $10 \mathrm{MeV}$, the influence of pion condensation on the nuclear equation of state strongly depends on the normal equation of state. If the energy per particle rises too fast, i.e., if the ground state compression constant is larger than 100-150 $\mathrm{MeV}$, no secondary minimum occurs. Even a van der Waals type behavior may be suppressed. Moreover, we have disregarded in the present consideration the destructive influence of the spinspin and isospin-isospin interactions $f_{0}^{\prime}$ and $g_{0}$ which will inhibit condensation appreciably. The critical density may be shifted to $\rho_{\mathrm{cr}} \sim 8 \rho_{0}$. At the se high densities the influence of pion condensation on the nuclear equation of state may be completely negligible. We therefore conclude that the nuclear equation of state alone may not give conclusive evidence of the condensation. Beyond that one must look for other anomalies connected with the second order phase transition, e.g., in the specific heat.

We have also investigated the temperature dependence of the critical density. We find for reasonable values of $\tilde{\beta}$ that the phase transition curve lies mostly within or even below the shock wave region, i.e., the stability of the pion condensate cannot be definitely claimed.

Nevertheless precritical phenomena, ${ }^{8}$ which do not need a fully established condensation, may occur. These phenomena are a keystone in the justification of macroscopic concepts, e.g., hydrodynamics in the description of high energy heavy ion collisions. It may be possible to study the relevance of a phase transition even if it does not show up the equation of state. The investigation of these secondary effects is therefore of great importance.
${ }^{1}$ H. Stöcker, J. Hofmann, J. A. Maruhn, and W. Greiner, Erice Lectures 1979 (edited by D. Wilkinson) for general reference.

${ }^{2}$ G. E. Brown and W. Weise, Phys. Rep. 27C, 1 (1976) and references therein.

${ }^{3}$ T. D. Lee and G. C. Wick, Phys. Rev. D 9, 2291 (1974).

${ }^{4}$ A. B. Migdal, Zh. Eksp. Teor. Fiz. $61, \overline{2209}$ (1971)
[Sov. Phys, -JETP 34, 1184 (1972)].

${ }^{5}$ A. B. Migdal, Rev. Mod. Phys. 50, 107 (1978).

${ }^{6}$ R. F. Sawyer and D. J. Scalapino, Phys. Rev. D $\underline{7}, 953$ (1973).

${ }^{7}$ V. Ruck, M. Gyulassy, and W. Greiner, Z. Phys. A277, 391 (1976).

${ }^{8}$ M. Gyulassy and W. Greiner, Ann. Phys. (N. Y.) 109, 
485 (1977).

${ }^{9}$ D. K. Campbell, R. F. Dashen, and J. T. Manassah, Phys. Rev. D 12, 979 (1975); 12, 1010 (1975); J. M. Irvine, Rep. Prog. Phys. 38, 1385 (1975).

${ }^{10}$ S. A. Moszkowski and C. G. Källman, Nucl. Phys. A287, 495 (1977); F. Boguta and A. R. Bodmer, ibid. $\underline{\underline{A 292}}, 413$ (1977); J. Boguta, Argonne National Laboratory report (unpublished).

${ }^{11}$ L. D. Landau, Zh. Eksp. Teor. Fiz. 32, 59 (1957) [Sov. Phys. -JETP $\underline{5}, 101$ (1957)]; ibid. 35, 97 (1958) [ibid. $\underline{8}$, 70 (1959)].

${ }^{12}$ A. B. Migdal, Theory of Finite Fermi Systems and Applications to Atomic Nuclei (Interscience, New York, 1967).
${ }^{13}$ M. R. Anastasio and G. E. Brown, Nucl. Phys. A285, 516 (1977).

${ }^{14}$ R. D. Mattuck, A Guide to Feynman Diagrams in the Many Body Problem (McGraw-Hill, New York, 1976).

${ }^{15}$ R. D. Mattuck and B. Johansson, Adv. Phys. 17 , 509 (1968).

${ }^{16}$ A. Fetter and J. D. Walecka, Quantum Theory of ManyParticle Systems (McGraw-Hill, New York, 1971).

${ }^{17} \mathrm{~S}$. O. Bäckman and W. Weise, Phys. Lett. 55B, 1 (1975).

${ }^{18}$ W. Scheid and W. Greiner, Z. Phys. 226, 365 (1969).

${ }^{19}$ L. D. Landau and E. Lifshitz, Statistical Physics (Pergamon, New York, 1960). 